Molina Saorín, Jesús; Nunes Corredeira, Rui Manuel (2012). La percepción social de los futuros maestros sōbre las personas con síndrome de Down: aplicación de la escala EPSD-1. Revista de Investigación Educativa, 30 (2), 383-396.

\title{
LA PERCEPCIÓN SOCIAL DE LOS FUTUROS MAESTROS SOBRE LAS PERSONAS CON SÍNDROME DE DOWN': APLICACIÓN DE LA ESCALA EPSD-I
}

\author{
Jesús Molina Saorín \\ Universidad de Murcia \\ Rui Manuel Nunes Corredeira \\ Universidade do Porto (Portugal)
}

\section{RESUMEN}

Las actitudes que siempre han despertado las personas con síndrome de Down han estado marcadas, principalmente, por la idea de irracionalidad y rechazo (Cáceres, 2004). Consecuentemente, han sido percibidas como un estorbo para la sociedad. Sin lugar a dudas, la influencia educativa de los maestros se convierte en una piedra angular para iniciar el cambio hacia otra conciencia social. En este sentido, hemos realizado una investigación en la cual analizamos la percepción social de los estudiantes de Magisterio ante diferentes aspectos de la vida de las personas con síndrome de Down. Ofrecemos los resultados extraídos de la aplicación de la Escala de Percepción Social hacia las personas con síndrome de Down (EPSD-1), utilizando como muestra a los alumnos del último curso de Educación Física (Universidad de Murcia; curso 2009/2010).

Para finalizar, hacemos hincapié en la necesidad de analizar la relación entre la percepción social y el plan de estudios que han cursado.

Palabras clave: Síndrome de Down; Percepción Social; Formación del Profesorado; Diversidad del Alumnado.

\footnotetext{
Correspondencia:

Jesús Molina Saorín (jesusmol@um.es); Rui Manuel Nunes Corredeira (rcorredeira@fade.up.pt)

1 Esta investigación ha sido financiada por el Ministerio de Educación, la Fundación para el síndrome de Down (FUNDOWN), la Fundación Séneca y la Fundación Cultural Privada Esteban Romero. Deseamos expresar nuestro agradecimiento a todas estas instituciones, junto a los alumnos de la Facultad de Educación de la Universidad de Murcia, sin los cuales esta investigación no hubiera sido posible.
} 


\title{
FUTURE TEACHERS' SOCIAL PERCEPTION OF PEOPLE WITH DOWN SYNDROME: APPLICATION OF THE EPSD-I SCALE
}

\begin{abstract}
Attitudes towards people with Down Syndrome (DS) have always been related to the idea of irrationality and rejection (Cáceres, 2004). People with DS have, therefore, been perceived as a nuisance to society. The educational influence of teachers is, no doubt, an essential element to initiate changes towards social awareness. In this respect, we carried out a study in which we analyzed the social perception of students of Education of different aspects of life of people with DS. In this article we present the results collected by means of the Social Perception Scale towards people with DS (EPSD-1) using final year students of Physical Education as a sample group (University of Murcia, Spain, during 2009/2010).

We highlight the need to examine the relationship between social perception and course curriculum.
\end{abstract}

Keywords: Down Syndrome; Social Perception; Teacher Training; Student Diversity.

\section{INTRODUCCIÓN}

Durante los últimos años, hemos asistido a ciertos cambios en la población con discapacidad que, según la EDAD (Encuesta sobre discapacidad, autonomía y dependencia -2008) resultan muy interesantes. Al parecer, la distribución de las personas con discapacidad no sigue un patrón aleatorio, sino que se concentra en unas zonas y no en otras, provocando cambios en la estructura demográfica. De la lectura de este informe (EDAD, 2008) se desprende que resulta necesario indagar sobre la naturaleza de estos datos, con objeto de conocer en qué medida podrían resultar condicionantes factores tales como la salud, los aspectos sanitarios, socio-económicos e, incluso, la percepción de la sociedad y los aspectos educativos. Durante los últimos años, la sociedad ha dado pasos importantes en su concepción acerca de la discapacidad y su entorno (Candel, 2003). Poco a poco, se van analizando viejos prejuicios que estereotipan y califican a quienes tienen alguna discapacidad, tildándoles de menos capaces que quienes no la tienen (Ebbeck y Weiss, 1998; Marsh y Hattie, 1996). Vamos incorporando, en nuestra percepción de la realidad, la idea de que todas las personas somos iguales, independientemente de que tengamos discapacidad o no, del sexo con el que nazcamos, de nuestra condición social, orientación sexual, religión, etcétera (García Fernández, 1995). Todos los avances sociales han tenido (y tienen) su reflejo en el lenguaje, que se convierte en el reflejo de las personas, y la sociedad que lo utiliza describe las especificidades de cada grupo social, mostrando sus concepciones en cada momento. Pero no debemos desviar nuestra atención. Las actitudes que siempre han despertado las personas con síndrome de Down -en todos los sectores sociales- han estado marcadas, principalmente, por la idea de irracionalidad y rechazo (Cáceres, 2004). Consecuentemente, estas personas han sido percibidas como un estorbo para la sociedad. Todo ello justifica las continuas oscilaciones de una actitud colectiva que gravita entre la tolerancia y la reprobación, la integración y la segregación. 
A pesar de que las transformaciones sociales y culturales han ido modificando las representaciones colectivas sobre la discapacidad intelectual, las antiguas concepciones siempre han subsistido, en cierta medida, entremezcladas con los nuevos conocimientos científicos y con nuevas formas semánticas de referirnos al mismo hecho. Sabemos que muchas personas mantienen una actitud negativa hacia las personas con síndrome de Down (Glenn y Cunningham, 2001), y que dicha actitud tiene un efecto perjudicial en la vida de estas personas con discapacidad (Madariaga, 2008). Y lo que resulta más importante, hemos experimentado el efecto de malas actitudes a través de los escasos niveles de interés, apoyo y compromiso social que encontramos, frecuentemente, con relación a este particular (Molina e Illán, 2011). En ese sentido, para promover actitudes positivas de la sociedad ante las personas con discapacidad intelectual, debemos superar el miedo sutil (pero penetrante) y la falta de comprensión, que resultan tan comunes en la sociedad actual. La idea de incluir a las personas con síndrome de Down (SD) en todos los aspectos de la vida cotidiana es, continuamente, desafiada por las actitudes negativas y estereotipos de una sociedad hegemónica (Jiménez, 2001; Illán, 2004). Tanto es así, que las actitudes y expectativas de la sociedad determinan, en gran medida, el grado hasta el cual las personas con este síndrome pueden aprender, desarrollar, amar, vivir o trabajar. Aunque la inclusión, por lo menos como filosofía, se ha vuelto ampliamente aceptada (véase Faria, 2005; Gilson, Cooke y Mahoney, 2005; Stone y May, 2002), la aceptación total de las personas con SD bien pareciera no haberse logrado aún. Varios autores (Fullana, Pallisera y Vilà, 2003; Peralta, González y Sobrino, 2005) ponen de manifiesto la preocupación por conocer cuál es la percepción que los estudiantes del título de Maestro, en general, mantienen hacia las personas con SD y otras discapacidades intelectuales. Por ello, con su actividad docente, tal vez legitimen y multipliquen entre sus alumnos tales percepciones. $\mathrm{Y}$, en particular, los de la especialidad de Educación Física, precisamente porque su actividad profesional futura está fuertemente ligada a este colectivo en lo referido a la actividad física no formal, al tiempo que también conlleva un marcado carácter educativo. Como destacan varios autores (véase García Pérez et al., 2010; Méndez García, 2009), los estudiantes de Magisterio (Educación Física) que están a punto de graduarse, constituyen un grupo con gran potencial para influir en la sociedad por su otro ámbito de actuación profesional vinculado al deporte y la actividad física. De ahí la importancia en conocer su percepción sobre diferentes aspectos sociales. Su trato directo con las personas con SD durante el ejercicio de su profesión (tanto en el entorno formal como no formal), les convierten en buenos indicadores de la concepción que sostiene el profesorado (en general), con respecto a las diferentes categorías de aquello que percibimos y pensamos a tenor de las personas con SD (Molina y Marques, 2009).

Como complemento a esta perspectiva Gómez del Valle et al. (2003) destacan la importancia de analizar el contenido de los planes de estudios ofertados en la universidad, por su marcada influencia en la configuración de tales percepciones sociales entre los universitarios. Es decir, si bien es posible iniciar unos estudios universitarios teniendo una percepción concreta de las personas con síndrome de Down y graduarse manteniendo tal percepción, diferentes autores (Moreno, Rodríguez, Saldaña y Aguilera, 2006) han demostrado que el tránsito por la universidad puede modificar tales percepciones durante el proceso de formación del alumnado. 
Parece razonable pensar que, con independencia de las causas que originen una percepción concreta hacia las personas con SD, si las opiniones de nuestros alumnos universitarios que están a punto de graduarse como Maestros resultasen (por citar un ejemplo) contrarias a la vida autónoma de las personas con SD, ciertamente podríamos plantearnos la necesidad de incorporar algún cambio en los planes de estudios que corrigiese tal percepción.

Por este motivo, en este trabajo hemos concentrado nuestro interés en analizar varios aspectos concretos, susceptibles de ser mensurados. En este sentido, y en consonancia con los autores citados, hemos realizado una investigación entre los estudiantes universitarios del título de Maestro en Educación Física de la Universidad de Murcia (curso 2009/2010), abordando el siguiente problema de estudio: conocer las opiniones $\mathrm{y}$ actitudes que tales estudiantes mantienen ante diferentes aspectos de la vida de las personas con síndrome de Down, relacionados con su integración social, autonomía, aceptación o sexualidad, entre otros.

\section{METODOLOGÍA DE LA INVESTIGACIÓN}

\section{Diseño}

En cuanto al diseño del estudio, hemos realizado un análisis factorial confirmatorio de los 10 factores presentes en la escala EPSD-1 (escala de percepción social hacia las personas con $\mathrm{SD}$ ), que recoge - precisamente- la percepción de los estudiantes de Magisterio hacia las personas con SD (Molina, Corredeira y Vallejo, en prensa). Hemos aplicado como solución factorial la rotación varimax, según la cual es posible reducir el número de variables que presentan saturaciones elevadas en cada factor, minimizando -de este modo- la interpretación de los resultados. Los criterios utilizados en la solución factorial han sido, en primer lugar, el número total de factores y, en segundo, destacar aquellos cuyo eigenvalue fuese superior a 1.

\section{Muestra}

El muestreo es de tipo censal, siendo la muestra de este estudio de 114 sujetos, que representan el $94 \%$ de la población, constituida por estudiantes de Magisterio de la Universidad de Murcia (especialidad de Educación Física), quienes se graduaron el curso pasado. El número total de sujetos es el resultado de la eliminación de aquellos alumnos cuyo instrumento de recogida de datos contenía errores y datos perdidos, teniendo finalmente un nivel de confianza superior al 97\%. En su conjunto, el 59\% son mujeres y el resto hombres (41\%). En cuanto a la edad, encontramos tres grandes categorías: el $47 \%$ tienen entre 20 y 21 años; un 33\% entre 22 y 25 años y el 20\% restante tienen entre 26 y 35 años. La inmensa mayoría son de nacionalidad española $(98,2 \%)$, con presencia también de nacionalidad iberoamericana $(0,9 \%)$ y otras nacionalidades $(0,9 \%)$. 


\section{Instrumento}

La escala EPSD-1 se nutre de una amplia información bibliográfica referida a aquellos ámbitos relacionados con la integración social y calidad de vida de las personas con SD (véase Arias, 2004; Molina e Illán, 2010), junto con trabajos referidos a la percepción social de la discapacidad (véase Bernardo y Matos, 2003; Corredeira, Corte-Real, Dias, Silva, y Fonseca, 2007; Crocker, Eklund y Kowalsky, 2000; Finlay y Lyons, 2000). Se trata de una escala de tipo Likert con cinco opciones (1-Totalmente de acuerdo; 2-De acuerdo; 3-Indiferente; 4-En desacuerdo; 5-Totalmente en desacuerdo). Está formada por 31 ítems organizados, aleatoriamente, en los diez factores siguientes: Exclusión social o marginación de las personas con SD (F1); rechazo de su autonomía e independencia (F2); rechazo a mantener relaciones afectivo/sexuales con personas con SD (F3); aceptación social en el ámbito educativo (F4); aceptación a tener descendencia con SD (F5); control externo de la sexualidad (F6); rechazo de la integración de las personas con SD (F7); control anticonceptivo (F8); proteccionismo (F9) y escasa formación docente y actuación paternalista ante la integración de personas con SD (F10). Por su parte, el reparto de variables entre los factores se organiza de la siguiente manera: F1 (10 variables); F2 (5 variables); F3, F4, F5, F6, F7, F8, F9 y F10 con 2 variables diferentes (cada factor). La EPSD-1 arroja un nivel de confianza superior al 98\% (según el análisis con STATS). En su conjunto, el instrumento original alcanzó un valor en el coeficiente alfa de 0,871 para la escala, hecho que le otorga una elevada fiabilidad (George y Mallery, 1995).

\section{Procedimiento}

Para la aplicación del instrumento, solicitamos el premiso oportuno a las autoridades académicas responsables. Una vez concedido, el procedimiento consistió en imprimir y distribuir los cuestionarios entre los alumnos durante una sesión presencial en el aula, utilizando unos diez minutos para ello y un único evaluador. Se trataba de un documento impreso de lectura óptica, cuyo vaciado de la información lo realizaría - posteriormente - un escáner. Por otra parte, y con el objetivo de asegurar un elevado nivel de confianza en los resultados, realizamos una versión digital del cuestionario, con el propósito de que todos aquellos estudiantes que no hubiesen podido asistir el día de la aplicación presencial, tuviesen la oportunidad de realizarlo y hacérnoslo llegar. Para la elaboración de este instrumento digital, contamos con el asesoramiento del Servicio de Apoyo a la Investigación de la Universidad de Murcia. El resultado fue un cuestionario que permitía rellenarlo desde una plataforrma digital (a la cual se accedía por invitación personal al correo institucional de la universidad) y recibirlo en tiempo real para su vaciado. Una vez en nuestra base de datos, se generaba un informe automático de respuesta con la información contenida.

\section{RESULTADOS}

A continuación, presentamos los resultados correspondientes a cada una de las subescalas (factores) pertenecientes a la aplicación de la EPSD-1. 
TABLA I

ESTADÍSTICOS DESCRIPTIVOS DE LAS ESCALAS

\begin{tabular}{|l|l|c|c|c|}
\hline \multicolumn{1}{|c|}{ Escala } & N & Media & Desv. Típ. \\
\hline $\mathbf{1}$ & $\begin{array}{l}\text { Exclusión social o marginación de las personas con } \\
\text { SD }\end{array}$ & 114 & $\mathbf{4 , 1 9}$ & 0,62 \\
\hline $\mathbf{2}$ & $\begin{array}{l}\text { Rechazo de la autonomía e independencia de las } \\
\text { personas con SD }\end{array}$ & 114 & 3,27 & 0,51 \\
\hline $\mathbf{3}$ & $\begin{array}{l}\text { Rechazo a mantener relaciones afectivo/sexuales } \\
\text { con personas con SD }\end{array}$ & 110 & 2,73 & 1,08 \\
\hline $\mathbf{4}$ & Aceptación social en el ámbito educativo & 113 & 3,75 & 0,90 \\
\hline $\mathbf{5}$ & Aceptación a tener descendencia con SD & 114 & 2,95 & 0,71 \\
\hline $\mathbf{6}$ & Control externo de la sexualidad & 114 & $\mathbf{2 , 4 3}$ & 0,94 \\
\hline $\mathbf{7}$ & Rechazo de la integración de las personas con SD & 113 & 2,64 & 0,81 \\
\hline $\mathbf{8}$ & Control anticonceptivo de las personas con SD & 113 & 3,32 & 0,68 \\
\hline $\mathbf{9}$ & Proteccionismo hacia las personas con SD & 114 & 3,18 & 0,69 \\
\hline $\mathbf{1 0}$ & $\begin{array}{l}\text { Escasa formación docente y actuación paternalista } \\
\text { ante la integración de personas con SD }\end{array}$ & 113 & 2,72 & 0,80 \\
\hline
\end{tabular}

La media más alta se ha obtenido en la subescala F1 de “Exclusión social o marginación de las personas con SD" (media $=4,19$; DT =0,62). Por el contrario, la media más baja se ha obtenido en la subescala F6 de "Control externo de la sexualidad" (media = $2,43$; DT $=0,94)$. Entre ambas subescalas, el resto de valores más elevados los alcanzan los factores 8, 2, 9 y 4, que obtienen puntuaciones medias entre los 3,18 (factor 8) y 3,75 valores (factor 4), con una desviación típica que va desde 0,51 a 0,90 (tal y como apreciamos en la Tabla II). Por último, los valores inferiores los reciben los factores $7,10,3$ y 5, alcanzando unas puntuaciones medias que oscilan entre los 2,64 valores (factor 7) hasta 2,95 (factor 5), con una desviación típica que oscila entre 0,71 y 1,08. El análisis factorial determina que la subescala F1 (Exclusión social o marginación de las personas con SD) se asocia de forma positiva con las siguientes subescalas (F2, F3 y F4) arrojando los siguientes valores:

- F2 (Rechazo de la autonomía e independencia de las personas con SD) ( $r=0,201$; $\mathrm{p}=0,032)$.

- F3 (Rechazo a mantener relaciones afectivo/sexuales con personas con SD) ( $\mathrm{r}=$ $0,295 ; p=0,002)$.

- F4 (Aceptación social en el ámbito educativo) $(r=0,437 ; p=0,000)$.

Por otra parte, la subescala F2 (Rechazo de la autonomía e independencia de las personas con SD), se encuentra positivamente relacionada con la subescala F3 (Rechazo a mantener relaciones afectivo/sexuales con personas con SD) $(r=0,600 ; p=0,026)$. Además, la subescala F3 (Rechazo a mantener relaciones afectivo/sexuales con personas con $\mathrm{SD})$ se encuentra directamente asociada a F4 (Aceptación social en el ámbito educativo) $(\mathrm{r}=0,359 ; \mathrm{p}=0,007)$. También hemos encontrado una relación directa y significativa 
entre los factores F3 (Rechazo a mantener relaciones afectivo/sexuales con personas con SD), F5 (Aceptación a tener descendencia con SD) ( $r=0,311 ; p=0,007), F 4$ (Aceptación social en el ámbito educativo) ( $\mathrm{r}=0,321 ; \mathrm{p}=0,01)$ y F10 (Escasa formación docente y actuación paternalista ante la integración de personas con SD) $(r=0,368 ; \mathrm{p}=0,005)$.

Asimismo, se evidencia una relación positiva entre F4 (Aceptación social en el ámbito educativo) y F9 (Proteccionismo hacia las personas con SD) $(\mathrm{r}=0,233 ; \mathrm{p}=0,001$ ), así como entre F6 (Control externo de la sexualidad) y F8 (Control anticonceptivo de las personas con SD) $(\mathrm{r}=0,281 ; \mathrm{p}=0,003)$. Por último, indicar una relación directa y significativa entre F7 (Rechazo de la integración de las personas con SD) y otras tres subescalas (F9, F8 y F10):

- F8 (Control anticonceptivo de las personas con SD) $(r=0,430 ; p=0,01)$.

- F9 (Proteccionismo hacia las personas con SD) $(r=0,322 ; p=0,001)$.

- F10 (Escasa formación docente y actuación paternalista ante la integración de personas con SD) $(r=0,380 ; p=0,000)$.

Desglosando estos resultados de cada uno de estos factores, subrayar que únicamente la subescala F1 (Exclusión social o marginación de las personas con SD) (media $=4,19$; DT $=0,62)$, ha alcanzado elevadas puntuaciones medias referidas a la actitud favorable hacia la integración social de las personas con SD. En segundo lugar, destacan las subescalas F2 (de Rechazo de la autonomía e independencia de las personas con SD), F8 (de Control anticonceptivo de las personas con SD) y F9 (de Proteccionismo hacia las personas con SD) cuyas puntuaciones medias alcanzadas se sitúan, fundamentalmente, en el terreno de la valoración media (65,5\%, también interpretada como posible neutralidad o desconocimiento), recogiendo valores salpicados por la actitud favorable (7\%) y también la desfavorable (27,5\%). En concreto, de la subescala F2 destacamos que un 18,4\% considera que las personas con SD no están preparadas para vivir en pareja; un $67,5 \%$ considera que no tienen derecho a decidir cómo o con quién pueden vivir. Dentro de este mismo factor, el 28,1\% están de acuerdo en que no debería permitírseles adoptar niños (el 30,7\% contestó con la puntuación intermedia), aspecto que viene de la mano de un $20,2 \%$ que considera que las personas con SD no están preparadas para educar o cuidar niños. Por su parte, la subescala F8 recoge una serie de ítems favorables al uso de anticonceptivos irreversibles para las personas con SD $(11,4 \%)$, o anticonceptivos eficaces $(29 \%)$, hecho que, inconscientemente, nos ha hecho transportarnos en el tiempo hasta la época de la alarma eugenésica (1912). Por último, la subescala F9 refleja que el empleo protegido es la única salida profesional para las personas con SD en tan solo un 5,3\% y, al mismo tiempo, que el hecho de tener un hijo con SD no condicionaría nuestra vida (71\%).

La subescala F7 (Rechazo de la integración de las personas con SD), también nos indica que les incomodaría la presencia de personas con SD en el mismo ámbito laboral $(16,6 \%)$ y, al mismo tiempo, que no está bien considerada (a nivel social) la vida independiente de estas personas (77,3\%). Siendo esto así, igualmente nos intranquiliza cómo podrá ser el desempeño profesional de estos futuros maestros, si se sienten incómodos cuando se les acerca una persona con SD, teniendo en cuenta que durante su ejercicio profesional estarán, necesariamente, obligados a trabajar con estos alumnos. La escala F10 (Escasa formación docente y actuación paternalista ante la integración de personas con $\mathrm{SD}$ ), recoge el acuerdo de los sujetos de nuestro estudio, con respecto a la 
consideración de que los profesores no están preparados para atender a los niños con SD $(79,8 \%)$ y, también, a la existencia de un sentimiento de pena entre el profesorado y hacia los niños con SD (30,7\%), por el cual los primeros facilitan las tareas para que estos alumnos aprueben (Molina y Marques, 2009). La subescala F3 (Rechazo a mantener relaciones afectivo-sexuales con personas con SD), nos indica que los maestros de nuestro estudio nunca sentirían deseo sexual por una persona con SD $(73,7 \%)$, ni tampoco se podrían enamorar de ella $(56,1 \%)$.

Acercándonos al final de este detallado análisis, el factor 5 (Aceptación a tener descendencia con SD) recoge los mensajes favorables a utilizar la opción del aborto en el caso individual de saber, previamente, que se está esperando un bebé con SD $(48,7 \%)$, o también el parecer favorable de los maestros a abortar en el caso de saber que sus padres están esperando un niño (futuro hermano del maestro) con este síndrome (51,8\%). Seguramente, este factor ofrece una información muy valiosa ante la consideración social negativa de las personas con SD, hecho que está en consonancia con los últimos factores que hemos presentado debidamente agrupados. Por último, el factor F4 (Aceptación social en el ámbito educativo) está directamente enraizado con la profesión docente. En concreto, la media de este factor $(3,75 ; \mathrm{DT}=0.9)$ nos indica que estos futuros maestros consideran que los niños con SD no deberían estudiar en escuelas ordinarias (57,9\%). Tan solo un 14,9\% se muestran favorables a esa inclusión educativa. Del mismo modo, dentro de este factor se recoge la convicción de que la presencia de alumnos con SD en las aulas, no contribuye al enriquecimiento del resto de los alumnos $(67,6 \%)$.

Tal y como ya hemos comentado, existe correlación entre el factor F1 y las subescalas F2, F3 y F4, de forma que cuanto mayor es la valoración de exclusión social o marginación de las personas con SD (F1), mayor es también el rechazo de la autonomía e independencia de estas personas, así como el rechazo a mantener relaciones afectivo/ sexuales con ellas y su aceptación social en el ámbito educativo. Hemos comprobado que los factores F2, F3 y F4 tienen gran presencia en cuando a las correlaciones con otros factores, de modo que parece que la autonomía, la sexualidad y la integración educativa se convierten en tres elementos clave a la hora de configurar la percepción social hacia el síndrome de Down. En este sentido, la subescala F2 también se encuentra positivamente relacionada con F3, confirmando así el presupuesto anterior. Del mismo modo, la subescala F3 se encuentra directamente asociada con F4, reforzando así la consistencia entre ambos factores. Además, la subescala F3 conserva una relación directa y significativa con los factores F5, F4 y F10, lo que supone que a mayor rechazo a relacionarse con personas con $\mathrm{SD}$, menor aceptación ante una posible descendencia de hijos con SD, menor aceptación social en el ámbito educativo y mayor creencia en la falta de preparación docente para trabajar con alumnos con este síndrome.

Por su parte, la subescala F4 aparece positivamente relacionada con F9, al igual que sucede entre los factores F6 y F8. Por lo tanto, la aceptación o rechazo social condiciona la percepción sobre el control anticonceptivo de las personas con SD. Del mismo modo, la valoración del control externo de la sexualidad incide en la percepción de proteccionismo hacia las personas con SD. Para finalizar, también hemos encontrado una relación directa y significativa entre F7 y las subescalas F8, F9 y F10, que nos indica que cuando los sujetos se posicionan rechazando la integración de las personas con 
SD, también lo hacen a favor del control anticonceptivo, a favor del proteccionismo y destacando una escasa formación docente y una marcada actitud paternalista hacia las personas con SD. Por lo tanto, podemos decir que existen seis grandes factores que, de algún modo, se interrelacionan hasta configurar esa percepción de esta muestra hacia las personas con SD (F1, F2, F3, F4, F6 y F7).

Con objeto de comprobar la posible relación existente entre la edad (entendida como variable categórica) y las subescalas contenidas en la EPSD-1, hemos realizado la prueba F de Anova. A partir de los resultados de esta aplicación, se desprende que ninguno de los contrastes realizados arroja un resultado significativo, por lo que no podemos considerar que la percepción social hacia las personas con SD esté relacionada con la edad. Asimismo, hemos aplicado la prueba t de Student para analizar la relación entre el sexo y las puntuaciones obtenidas en la EPSD-1. Hemos encontrado una relación significativa entre el sexo y la escala F1 de Exclusión social o marginación de las personas con SD $[t(53,315)=-2,030 ; p=0,047]$, siendo la puntuación de las mujeres superior a la de los varones. En la subescala F4 de Aceptación social en el ámbito educativo, también hemos encontrado una asociación con el sexo $[\mathrm{t}(111)=-2,375 ; \mathrm{p}=0,019]$, siendo igualmente superior la puntuación de las mujeres, como en el caso anteriormente comentado (F1). Del mismo modo, y siguiendo esa misma tendencia femenina, en la escala F7 de Rechazo de la integración de las personas con $\operatorname{SD}[\mathrm{t}(111)=-2,030 ; \mathrm{p}=0,045]$, la puntuación de las mujeres ha sido superior. Por último, también se ha encontrado que la puntuación de las mujeres es superior en la escala F8 de Control anticonceptivo de las personas con SD $[\mathrm{t}(111)=-2,718 ; \mathrm{p}=0,008]$.

\section{DISCUSIÓN}

Al parecer, nos encontramos ante un tema de investigación ciertamente polémico. Reflexionar sobre la inclusión o exclusión de las personas con síndrome de Down, valorando el modo en que estas pueden y deben vivir, sentir o amar es, sin lugar a dudas, un asunto que no deja impasible a la población (Crabtree y Rutland, 2001; Crocker, Eklund y Kowalsky, 2000). Cuando hablamos de cuestiones relacionadas con determinados ámbitos de la vida social, económica o, incluso, política, encontramos sectores de población reacios a emitir un juicio, en ocasiones por desconocimiento del asunto abordado, por la lejanía del mismo, por falta de empatía con el objeto de estudio o por incomodidad ante un posicionamiento firme al respecto. Sin embargo, cuando las preguntas se refieren a personas con SD y, en concreto, cuando circunscribimos las pesquisas al cuerpo de Maestros, descubrimos que existe siempre un posicionamiento polarizado. En este sentido, es muy difícil encontrarnos a un maestro que manifieste su indiferencia o falta de información (para responder) al respecto de cuestiones relacionadas con alumnos (o adultos) con este síndrome. Por algún motivo, preguntar por este colectivo no deja a los maestros indiferentes. En ese sentido, nos llama la atención que entre el colectivo de estudiantes existan actitudes que expresan el desagrado por compartir lugares de ocio con personas con SD (13,1\%). Un porcentaje similar $(14,1 \%)$ no participaría en un viaje organizado con personas con SD. En esta misma línea, un 20.2\% se siente incómodo cuando se le acerca una persona con SD; este porcentaje se acentúa cuando afirman que si una persona con SD se les acerca, no le dan atención o 
conversación (88,6\%). Dentro de este primer factor, también destaca un 5,3\% que considera que es necesario prohibir que las personas con SD tengan relaciones sexuales, junto con un $12,3 \%$ que considera que sí pueden tenerlas, pero únicamente con otras personas que también tengan este síndrome. Más de la mitad $(58,8 \%)$ consideran que, socialmente, la presencia de personas con SD incomoda, hecho que se ve reforzado con un $7,9 \%$ que confirman sentir vergüenza paseando por la calle junto a una persona con SD. Desde un punto de vista directamente relacionado con lo educativo, los valores más llamativos de este factor también serían el 7,1\% que declara que le molestaría que su hijo tuviese compañeros de clase con SD, junto al 14,9\% que preferiría no tener alumnos con SD en su aula. Pese a ser porcentajes pequeños (en ocasiones), nos sorprenden negativamente, puesto que entendemos que el paso por una facultad de educación y la obtención del título de Maestro, debería llevar de la mano la supresión total de determinado tipo de actitudes. Sin lugar a dudas, los datos ofrecidos por las subescalas F2, F8 y F9 despiertan nuestra curiosidad, en tanto en cuanto apreciamos que en la medida en que nos apartamos de aspectos genéricos como la integración social (F1), y nos adentramos en ámbitos de mayor intimidad (como los recogidos en F2, F8 y F9), las percepciones de nuestros alumnos inician su desplazamiento desde la actitud marcadamente favorable a la diversidad, hacia otra dominada por la neutralidad (o indiferencia). En este sentido, y siguiendo a diferentes autores (Čurdová, Vermeer y Válková, 2001; Scholtes, Vermeer y Meek, 2002; Vaughn, Hoga, Kouzekanani y Shapiro, 1990) consideramos tremendamente oportuno discutir sobre los porqués de tales respuestas, en la medida en que entendemos que un alumno de Magisterio que se gradúa, a juzgar por el planteamiento del título universitario, debería mostrar actitudes necesariamente favorables a los contenidos recogidos en las escalas F2, F8 y F9. Realmente, situarse dentro de ese terreno neutral (o incluso negativo) nos obliga a reflexionar y analizar la posible relación existente entre la percepción del alumno, antes de su ingreso en la facultad, y su evolución (si la hay) durante su proceso de aprendizaje hasta que titula. Y este cambio de polaridad en la respuesta, gravitando desde aquellas que demuestran actitudes consideradas como positivas hacia otras más neutrales, tiene también un componente antagónico, orquestado alrededor de respuestas dominadas por actitudes que entendemos como negativas y contrarias a lo que debería ser el perfil de Maestro, tal y como se recoge en las subescalas F3, F4, F5, F6, F7 y F10. Ordenadas en orden creciente (en función de la media), la subescala F6 (Control externo de la sexualidad), arroja un valor medio de 2,64, lo que nos indica que estos alumnos están de acuerdo $(68,5 \%)$ con que familiares y expertos realicen un control de la sexualidad (y relaciones sexuales) de las personas con SD.

El factor 10 pone de manifiesto las debilidades del sistema de formación universitario para los maestros, en la medida en que estos nos informan de una deficiencia en aspectos formativos cuya responsabilidad reside, directamente, en la universidad. Será, por lo tanto, muy interesante, tratar de averiguar en qué medida las variables asociadas a esta baja formación dependen del servicio ofrecido desde la universidad. Del mismo modo, el factor 3 ofrece una información muy rica, desde el punto de vista emocional, en la medida en que (al parecer) tener síndrome de Down supone una barrera de partida que coarta las posibilidades de un maestro para conocer y mantener una relación (afectiva y/o sexual) con una persona con SD. Nos preocupa que este pen- 
samiento esté afincado en el maestro, ya que al ser la educación una actividad cargada de valores (véase Hagger, Lindwall, y Hulya, 2004; Stone y May, 2002), posiblemente exista una relación directa entre su pensamiento y la visión que, sobre las personas con SD, pueda proyectar entre sus alumnos. El cuarto factor reviste una grandísima importancia, en tanto en cuanto pone en entre dicho la formación universitaria referida a todo lo relacionado con la diversidad educativa del alumnado, las necesidades educativas especiales y el proceso de integración e inclusión (Corredeira, 2001; Núñez y Jódar, 2010). Contenidos que, de manera troncal y obligatoria, son objeto de estudio en el plan académico del título de Maestro. Parece sensato pensar que, en el proceso formativo, exista algún tipo de variables capaces de generar un ruido tal que anule la consecución de buena parte de las competencias profesionales esperadas en el maestro.

\section{CONCLUSIONES}

Para finalizar, seguramente podemos decir que con esta investigación cobra vida el testimonio de aquellos que, en el momento actual, bien pudieran estar ejerciendo la profesión de Maestro en cualesquiera de los centros educativos (públicos o privados) diseminados por la geografía española, pues sin duda ya han alcanzado la licencia ministerial para tal desempeño. ¿Y qué nos puede ofrecer esta información? Evidentemente, no solo conocer el parecer de una promoción de maestros al respecto de las personas con SD, sino que también nos ofrece la oportunidad de reflexionar (como profesores universitarios e investigadores), ante la posible influencia entre el parecer final de los alumnos y el plan de estudios que han cursado durante su permanencia en la facultad.

Posiblemente, estos últimos años repletos de discursos educativos intencionadamente influenciados por borrascas europeas, anunciadoras de lluvias procedentes de los campos de Pisa y Bolonia, nos han obligado a cambiar los escenarios, los discursos y las prácticas docentes, como mínimo en el plano semántico e impreso en el que siempre gravitan nuestras intenciones (otra cosa será - esperamos analizarlo en breve- lo que realmente hemos hecho en las aulas). Con los datos aportados en nuestro estudio, disponemos de una valiosa información de un título universitario que, históricamente, ha desarrollado su labor profesional en escenarios muy próximos a los compartidos por las personas con SD. De algún modo, es nuestro deseo orientar nuestras futuras investigaciones a conocer la posibles relaciones existentes entre el plan de formación cursado por estos alumnos y sus posicionamientos que, a pesar de tener una constante personal (individual) marcada por la historia de vida saboreada en primera persona, también posee una dimensión didáctica, formativa, pedagógica y técnica depurada durante todo un proceso de - como diríamos ahora - capacitación y formación para un desarrollo competente de la profesión. Este será, sin dudas, un ámbito de investigación que ocupará nuestras mentes a partir de ahora.

\section{REFERENCIAS BIBLIOGRÁFICAS}

Arias, J. M. (2004). Introducción a la investigación en Ciencias Sociales. En A. Hidalgo y R. Medina (Eds.), Cooperación al desarrollo y bienestar social (pp. 87-115). Oviedo, España: Eikasia Ediciones. 
Bernardo, R. P., \& Matos, M. G. (2003). Adaptação portuguesa do physical self-perception profile for children and youth e do perceived importance profile for children and youth. Análise Psicológica, 2 (XXI), 127-144.

Cáceres, C. (2004). Sobre el concepto de discapacidad. Una revisión de las propuestas de la OMS. Revista electrónica de audiología, 2 (3), 74-77.

Candel, I. (2003). Elaboración del Programa de Atención Temprana. En I. Candel (Dir.), Atención Temprana. Niños con síndrome de Down y otros problemas del desarrollo (pp. 29-55). Madrid, España: FEISD.

Corredeira, R. (2001). Competência percebida e aceitação social em crianças com paralisia cerebral: tradução e adaptação da Pictorial Scale of Perceived Competence and Social Acceptance in Children with Cerebral Palsy. Oporto, Portugal: Facultad del Deporte de la Universidad de Oporto.

Corredeira, R., Corte-Real, N. J., Dias, C., Silva, M. A., \& Fonseca, A. M. (2007). Como avaliar a percepção de competência e aceitação social de crianças com paralisia cerebral? Estudo inicial para a determinação das propriedades psicométricas da versão portuguesa da Dutch Pictorial Scale of Perceived Competence and Social Acceptance in Children with Cerebral Palsy. Revista Brasileira de Educação Especial, 13 (3), 325-344.

Crabtree, J., \& Rutland, A. (2001). Self-evaluation and social comparison amongst adolescents with learning difficulties. Journal of Community y Applied Social Psychology, 11 (5), 347-359.

Crocker, P. R. E., Eklund, R. C., \& Kowalsky, K. C. (2000). Children's physical activity and physical self-perceptions. Journal Sport Sciences, 18, 383-394.

Čurdová, J., Vermeer, A., \& Válková, H. (2001). Measuring perceived and social acceptance in young children with cerebral palsy: the construction of a Czech Pictorial Scale. Gymnica, 1 (1), 27-36.

Ebbeck, V., \& Weiss, M. R. (1998). Determinants of children's self-esteem: Influence of perceived competence and affect. Pediatric Exercise Science, 10, 285-298.

Faria, L. (2005). Desenvolvimento do auto-conceito físico nas crianças e nos adolescentes. Análise Psicológica, 23 (4), 361-371.

Finlay, M., \& Lyons, E. (2000). Social categorizations, social comparisons and stigma: presentations of self in people with learning difficulties. British Journal of Social Psychology, 39, 129-146.

Fullana, J., Pallisera, M., \& Vilà, M. (2003). La investigación sobre los procesos de integración laboral de personas con discapacidad en entornos ordinarios. Un estudio de casos cualitativo. Revista de Investigación Educativa, 21 (2), 305-321.

García Fernández, J. L. (1995). Sexualidad y minusvalías psíquicas: la necesidad de saber. La Cristalera, 5, 44-48.

García Pérez, R., Rebollo, M. A., Buzón O., González R., Barragán, R., \& Ruíz, E. (2010). Actitudes del alumnado hacia la igualdad de género. Revista de Investigación Educativa, 28 (1), 217-232.

George, D., \& Mallery, P. (1995). SPSS/PC+ Step by step. A simple guide and reference. Belmont, CA: Wadsworth Publishing.

Gilson, N. D., Cooke, C. B., \& Mahoney, C. A. (2005). Adolescent physical self-perceptions, sport/exercise and lifestyle physical activity. Health Education, 105 (6), 437-450. 
Glenn, S., \& Cunningham, C. (2001). Evaluation of self by young people with Down Syndrome. International Journal of Disability, Development and Education, 48, 163-177.

Gómez del Valle et al. (2003). Identificación de los estilos de aprendizaje predominantes en estudiantes de Magisterio de la Facultad de Ciencias de la Educación de la Universidad de Cádiz. Revista Electrónica Interuniversitaria de Formación del Profesorado, 6 (2). Recuperado de http://aufop.com/aufop/uploaded_files/articulos/ 1227721086.pdf.

Hagger, M., Lindwall, M., \& Hulya, A. (2004). A cross-cultural evaluation of a multidimensional and hierarchical model of physical self-perceptions in three national samples. Journal of Applied Social Psychology, 34, 1075-1107.

Illán, N. (2004). Materiales para trabajar la autonomía y vida independiente de las personas con discapacidad psíquica. Madrid, España: FEISD.

Instituto Nacional de Estadística [INE] (2008). Encuesta sobre Discapacidades, Autonomía personal y situaciones de Dependencia. Madrid, España: Autor. Recuperado de http:// www.ine.es/jaxi/menu.do?type=pcaxisypath $=/ \mathrm{t} 15 / \mathrm{p} 418$ yfile $=$ inebasey $\mathrm{L}=0$

Jiménez, A. (2001). Encuesta sobre Discapacidades, Deficiencias y Estado de Salud (1999). Breve análisis de los primeros resultados. Recuperado de http://usuarios.discapnet.es/ AJimenez/encuesta/minusval.htm

Madariaga, A. (2008). Claves del acceso al ocio de toda la ciudadanía. En M. J. Monteagudo (Ed.), La experiencia de ocio: una mirada científica desde los estudios de ocio (pp.287-316). Bilbao, España: Universidad de Deusto.

Marsh, H. W., \& Hattie, J. (1996). Theoretical perspectives on the structure of selfconcept. En B. A. Bracken (Ed.), Handbook of self-concept. Developmental, social, and clinical considerations (pp. 38-90). Nueva York, NY: John Wiley \& Sons, Inc.

Méndez García, R. M. (2009). Los estudiantes: su percepción sobre lo que implica "ser estudiantes" y "hacer universidad". Revista de Investigación Educativa, 27 (1), 9-26.

Molina, J., \& Illán, N. (2010). Patrones de actuación del profesorado de Educación Infantil para la integración efectiva de los alumnos con síndrome de Down. Revista Iberoamericana de Educación, 52 (7), 3-11.

Molina, J., \& Illán, N. (2011). El proceso de integración efectiva de los alumnos con síndrome de Down en Educación Infantil: variables influyentes en dicho proceso. Revista Española de Pedagogía, 248, 5-22.

Molina, J., \& Marques, C. (2009). Experiencia docente en Educación Física y alumnos con necesidades educativas específicas: estudio de correlación. Retos. Nuevas tendencias en Educación Física, Deporte y Recreación, 16, 16-21.

Moreno, F. J., Rodríguez, I., Saldaña, D., \& Aguilera, A. (2006). Actitudes ante la discapacidad en el alumnado universitario matriculado en materias afines. Revista Iberoamericana de Educación, 40 (5). Recuperado de http://www.rieoei.org/ investigacion/1491Moreno.pdf

Núñez, J. P., \& Jódar, R. (2010). La integración socio-afectiva de los niños con síndrome de Down en aulas de integración y de educación especial. Revista de Educación, 353, 549-569.

Peralta, F., González, M. C., \& Sobrino, A. (2005). Creencias y conocimiento de los profesores acerca de la conducta autodeterminada. Revista de Investigación Educativa, 23 (2), 433-448. 
Scholtes, V., Vermeer, A., \& Meek, G. (2002). Measuring perceived competence and social acceptance in children with cerebral palsy. European Journal of Special Needs Education, 17 (1), 77-87.

Stone, C. A., \& May, A. J. (2002). The accuracy of academic self-perceptions in adolescents with learning disabilities. Journal of Learning Disabilities, 35, 370-383.

Vaughn, S., Hoga, A., Kouzekanani, K., \& Shapiro, S. (1990). Peer acceptance, selfperceptions and social skills of learning disabled students prior to identification. Journal of Educational Psychology, 82, 101-106.

Fecha de recepción: 3 de junio de 2011.

Fecha de revisión: 25 de julio de 2011.

Fecha de aceptación: 31 de octubre de 2011. 